\title{
Association of serum albumin levels with inflammation and clinical outcomes in children with acute bronchiolitis.
}

\author{
Moisés Rodríguez-González ${ }^{1}$, Patricia Rodríguez-Campoy ${ }^{1}$, Ana Estalella-Mendoza ${ }^{1}$, \\ Lorena Estepa-Pedregosa ${ }^{1}$, and J. Carlos Flores-González ${ }^{1}$ \\ ${ }^{1}$ Hospital Universitario Puerta del Mar
}

October 26, 2020

\begin{abstract}
Objective: To evaluate if hypoalbuminemia on admission predict disease severity in children with acute bronchiolitis (AB). Working hypothesis: Hypoalbuminemia is associated with worse outcome in infants with AB. Study design: Single-centre prospective cohort study. Patient-subject selection: Infants aged $<12$ month-old with AB. Methodology: Serum albumin levels were determined within the first 24 hours upon inclusion. The primary outcome was the need of pediatric intensive care unit (PICU) admission. Results: We enrolled 90 cases of AB. Serum albumin was independently associated with C-Reactive protein levels $(\mathrm{CRP})(\mathrm{rs}=-0,28 ; \mathrm{p}=0.002)$. Fourteen $(15.5 \%)$ cases required PICU admission. They presented lower serum albumin levels $(3.7(0.11)$ vs $4(0.5) \mathrm{g} / \mathrm{dl} ; \mathrm{p}=0.034)$ regarding those patients without severe illness. In the multivariate logistic regression analysis, hypoalbuminemia was independently associated with a higher risk of severe illness (adjusted Odds Ratio 4.1 $(1.2-85) ; \mathrm{p}=0.032)$. The area under the ROC curve for serum albumin to predict adverse outcome was 0.70 (95\% Confidence interval of 0.59-0.79). A cut-off point of $3.5 \mathrm{~g} / \mathrm{dl}$ presented a sensitivity of 0.71 , specificity of 0.68 , positive predictive value of 0.29 , and negative predictive value of 0.92 . Conclusion: Low serum albumin levels at admission are significantly associated with higher PICU admission rates in infants with AB. The inflammatory response could play a key role in the occurrence of hypoalbuminemia in AB.
\end{abstract}

\section{Title Page}

Type of article: Original article

Title: Association of serum albumin levels with inflammation and outcomes in children with acute bronchiolitis.

Authors: Moisés Rodríguez-González ${ }^{1,3}$, Patricia Rodríguez-Campoy ${ }^{2,3}$, Ana Estalella-Mendoza ${ }^{2,3}$, Lorena Estepa-Pedregosa ${ }^{2,3}$, J Carlos Flores-González ${ }^{2,3}$.

\section{Affiliations:}

(1) Pediatric Cardiology Division. Puerta del Mar University Hospital. Cádiz. Spain.

(2) Pediatric Intensive Care Unit. Puerta del Mar University Hospital. Cádiz. Spain

(3) Biomedical Research and Innovation Institute of Cádiz (INiBICA) Research Unit, Puerta del Mar University Hospital University of Cádiz, Spain.

Corresponding author: Ana Estalella Mendoza.Pediatric Intensive Care Unit. Puerta del Mar University Hospital. Avda Ana de Viya 21, 11009 Cádiz, Spain. E-mail:anaestalella@gmail.com. Telephone number: +34 956002256

Keywords: Acute bronchiolitis; Serum Albumin; Biomarkers; Inflammation 
Short title: Rodriguez-Gonzalez et al: Albumin and inflammation in acute bronchiolitis.

\begin{abstract}
Objective: To evaluate if hypoalbuminemia on admission predict disease severity in children with acute bronchiolitis (AB).
\end{abstract}

Working hypothesis: Hypoalbuminemia is associated with worse outcome in infants with AB.

Study design: Single-centre prospective cohort study.

Patient-subject selection : Infants aged $<12$ month-old with AB.

Methodology: Serum albumin levels were determined within the first 24 hours upon inclusion. The primary outcome was the need of pediatric intensive care unit (PICU) admission.

Results: We enrolled 90 cases of AB. Serum albumin was independently associated with C-Reactive protein levels $(\mathrm{CRP})\left(\mathrm{r}_{\mathrm{s}}=-0,28 ; \mathrm{p}=0.002\right)$. Fourteen $(15.5 \%)$ cases required PICU admission. They presented lower serum albumin levels $(3.7(0.11)$ vs $4(0.5) \mathrm{g} / \mathrm{dl} ; \mathrm{p}=0.034)$ regarding those patients without severe illness. In the multivariate logistic regression analysis, hypoalbuminemia was independently associated with a higher risk of severe illness (adjusted Odds Ratio $4.1(1.2-85) ; \mathrm{p}=0.032)$. The area under the ROC curve for serum albumin to predict adverse outcome was 0.70 (95\% Confidence interval of 0.59-0.79). A cut-off point of $3.5 \mathrm{~g} / \mathrm{dl}$ presented a sensitivity of 0.71 , specificity of 0.68 , positive predictive value of 0.29 , and negative predictive value of 0.92 . Conclusion:Low serum albumin levels at admission are significantly associated with higher PICU admission rates in infants with $\mathrm{AB}$. The inflammatory response could play a key role in the occurrence of hypoalbuminemia in $\mathrm{AB}$.

\title{
Main Text
}

\section{Introduction}

Acute bronchiolitis (AB) is the leading cause of lower respiratory infection and hospital admission among children up to 2 years of age worldwide ${ }^{1}$. Approximately 2-6\% cases of AB will develop a severe form of the disease, requiring admission at the pediatric intensive care unit (PICU) ${ }^{2}$. In absence of any definitive treatment, the major goal in $\mathrm{AB}$ is the prevention and early identification of infants at risk for develop a severe illness in order to provide the best management options and potentially decrease morbidity ${ }^{3}$. Current guidelines recommend the identification of specific risk factors (congenital heart disease (CHD), chronic lung disease (CLD), prematurity) and the clinical assessment as the best tools to asses severity, predict evolution and tailor the management accordingly ${ }^{4}$. However, most hospitalizations correspond to healthy infants, who can also develop a severe $\mathrm{AB}^{1,2}$, and most clinical scores for $\mathrm{AB}$ are not well validated and fail to predict outcomes $^{5}$. In this context, the identification of novel biomarkers with adequate predictive value for disease severity in $\mathrm{AB}$ is an area of increasing research ${ }^{6-8}$.

Albumin is a $69 \mathrm{kDa}$ protein that is mainly synthesized by the liver and plays an important role in a number of physiological mechanisms. It has long been well established that hypoalbuminemia is a powerful prognostic marker in the general population and many pathological settings, mainly as a result of malnutrition and inflammation ${ }^{9-13}$. During severe AB a reduced energetic intake, impaired nutritional status and an inflammation with a cytokine-mediated acute phase response can coexist, leading to lowered levels of serum albumin. However, the association between albumin and $\mathrm{AB}$ severity has been little investigated in this setting ${ }^{14}$.

The primary objective of this study was to evaluate if serum albumin levels on admission are associated with severity of illness in infants with $\mathrm{AB}$. A secondary objective was to explore possible causes of these low plasmatic levels in this setting.

\section{Materials and methods}

- Design, settings and study population: This was a single-centre prospective cohort study including infants aged less than 12 month-old evaluated at the Pediatric Department of our institution (a tertiary 
university-affiliated hospital in Spain) due to AB between October 1, 2018 and October 1, 2019. The diagnosis of $\mathrm{AB}$ was based on personal history, clinical symptoms and physical exam. The pediatrician in charge, who followed the recommendations of current $\mathrm{AB}$ guidelines, managed all patients. The exclusion criteria were patients previously diagnosed with malnutrition, a previous episode of $A B$, bacterial superinfection (based on blood or endotracheal cultures), patients that received any intravenous fluids including albumin before the intervention, and patients with incomplete intervention or medical records. Our Institutional Review Board approved the study. Informed consent was obtained for all patients.

- Intervention, Data Collection and Definitions: All patients underwent clinical, laboratory, and microbiologic evaluation. Characteristic data were recorded from medical records of the patients during hospitalization. The attending physician assessed clinical presentation. The bronchiolitis score of Sant Joan de Déu (BROSJOD) score ${ }^{15}$ was the clinical score used to assess the respiratory state severity at admission. A BROSJOD score greater than 10 points is indicative of severe clinical state. The blood collection was drawn within the first 24 hours upon inclusion of the patient in the study. Biochemical data including serum albumin levels were recorded. PCR analysis of nasopharyngeal mucus for respiratory viruses was routinely analyzed in all patients. Blood culture was performed only in cases with clinically suspected bacterial sobreinfection .

- Research outcome: The primary outcome were the need of PICU admission during the episode of AB, mortality and the length of stay (LOS) hospitalization. PICU admission criteria for AB in our institution are the presence of apnea, extreme bradycardia, need of respiratory support greater than high-flow nasal cannula oxygen therapy, or need of inotropic support. According to these data, patients that required PICU admission were classified as severe $\mathrm{AB}$ group.

- Statistical analysis: Continuous data are presented as median (range) or mean (standard deviation) after testing for normality with the Shapiro-Wilk test. Categorical data are presented as frequencies and percentage. Mean comparison was performed using Student's t test or Wilcoxon Mann-Whitney test as appropriate. Proportions were compared using Chi-square test or exact methods as necessary. Pearson and Spearman coefficients were used to assess correlations between continuous data. Multiple linear regression analysis was performed to identify variables independently associated with the dependent variable serum albumin. Only statistically significant variables in the correlation tests were entered into the multivariate analysis. Because the absence of clear normal range values for serum albumin in infants, the serum albumin level was dichotomized at the 25th percentile of the entire cohort and defined as hypoalbuminemia if the level was minor or equal of $3.5 \mathrm{~g} / \mathrm{dL}$. Multivariate logistic regression analysis was used to determine if hypoalbuminemia was independent predictor for adverse outcome. Significant variables detected in the univariate analysis and which were considered to be clinically relevant (known risk factors for severe AB) were entered into this multivariate analysis. Collinearity between variables was evaluated. The relative risks were expressed as odds ratios (OR) and 95\% confidence intervals (95\% CI). A receiver operator curve (ROC) analysis was used to determine the diagnostic accuracy of serum albumin for adverse outcome. The values of sensitivity, specificity, negative predictive value (NPV) and positive predictive value (PPV) were calculated for serum albumin cut-off point of $<3.5 \mathrm{~g} / \mathrm{dl}$. All the statistical analyses were performed using the Stata software (StataCorp. 2014. Stata Statistical Software: Release 14. College Station, TX: StataCorp LP.). A $\mathrm{P}$ value minor than 0.05 was considered statistically significant.

\section{Results}

- Baseline Data: A total of 107 infants aged 12 months or less with AB were assessed for eligibility. Of them, 101 patients accepted to participate, and we excluded 11 cases because of the presence of a previous $\mathrm{AB}$ episode (5) and bacterial superinfection (6), resulting in a sample size of 90 cases of $\mathrm{AB}$ (median age of 1 (0.5-7) months; 57 (60\%) male). The most prevalent causative agent was respiratory syncytial virus (RSV) (59 cases; 65\%). A total of 13 (14.5\%) of cases presented a known comorbidity associated to the development of a severe disease (1 case of congenital heart disease, 1 case of chronic pulmonary disease and 11 cases of prematurity). The patients consulted after 1 (0-7) days from the beginning of the symptoms, with a median BROSJOD score of $6(2-14)$ points. Up to $10(11 \%)$ patients were classified as severe clinical state accordingly with BROSJOD score $(>10)$ at admission. A total of $80(89 \%)$ cases required hospital 
admission with a median length of stay of 3 (0-19) days. Up to 14 (15.5\%) patients required PICU admission during a median of 6.5 (3-16) days. Regarding the respiratory support, a total of 55 (61\%) cases required oxygen therapy throw nasal cannula, $10(11 \%)$ cases required non-invasive ventilation (NIV), and $3(3.5 \%)$ cases required mechanical ventilation $(\mathrm{MV})$. No cases required inotropic support. There were no cases of mortality.Table 1 summarizes the baseline characteristics of the entire population and comparison between those with and without severe illness.

- Serum Albumin in infants with AB: The mean value of serum albumin in the entire group study was $4(0.47) \mathrm{g} / \mathrm{dl}$, and $15(16 \%)$ patients presented hypoalbuminemia (levels under $3.5 \mathrm{~g} / \mathrm{dl}$ ). The scatter plot of serum albumin displayed a significantly correlation with age $\left(\mathrm{r}_{\mathrm{s}}=0.50 ; \mathrm{p}<0.001\right)$, weight for age percentile $\left(\mathrm{r}_{\mathrm{s}}=0.33 ; \mathrm{p}=0.002\right), \mathrm{CRP}\left(\mathrm{r}_{\mathrm{s}}=-0.27 ; \mathrm{p}=0.008\right), \mathrm{pCO} 2\left(\mathrm{r}_{\mathrm{s}}=-0.32 ; \mathrm{p}=0.003\right)$, and $\mathrm{HCO} 3(\mathrm{r}=-0.24 ; \mathrm{p}=0.029)$ levels (Figure 1). Multiple-linear regression analysis confirmed the age, the weight for age percentile and CRP levels as the variables independently associated with serum albumin (Table 2). The patients with a severe $\mathrm{AB}$ presented lower serum albumin levels at admission than those patients without a severe illness $(3.7(0.11)$ $\mathrm{g} / \mathrm{dl}$ vs $4(0.5) \mathrm{g} / \mathrm{dl} ; \mathrm{p}=0.034)$ (Figure $2 \mathrm{~A}$ ). Those patients with serum albumin $<3.5 \mathrm{~g} / \mathrm{dl}$ presented a five-fold risk of PICU admission (OR 5.5 (CI95\% 1-55-19); $\mathrm{p}=0.008$ ). After adjusting for potential confounders (age, weight for age, presence of comorbidity, BROSJOD score, respiratory acidosis and CRP) in a multivariate logistic regression analysis, serum albumin level less than $3.5 \mathrm{~g} / \mathrm{dl}$ remained independently associated with a higher risk of severe $\mathrm{AB}(\mathrm{aOR} 4.1(1.2-24) ; \mathrm{p}=0.032)$. The ROC curve for serum albumin to predict a severe AB was generated, yielding an AUC of 0.70 (95\% CI 0.59-0.79) (Figure 3). A cut-off point of $3.5 \mathrm{~g} / \mathrm{dl}$ presented a sensitivity of 0.71 , specificity of 0.68 , positive predictive value of 0.29 , and negative predictive value of 0.92 . Finally, lower levels of serum albumin at admission correlated with the length-of-stay (LOS) hospitalization $(\mathrm{r}=-0.20 ; \mathrm{p}=0.036)$ (Figure 2B).

\section{Discussion}

In this observational prospective study involving 90 infants with $\mathrm{AB}$, an observational correlation has been observed between severity of illness and serum albumin levels; the children who had higher severity of illness, requiring PICU admission and longer hospitalizations had lower albumin levels at admission. Although the predictive value of hypoalbuminemia was only moderate, these results highlight a possible role for albumin as a biomarker for severity in this setting.

Serum albumin is considered as a marker of disease severity in critically ill adult patients, with low serum albumin concentrations being reported in $30 \%-50 \%$ of $\operatorname{cases}^{12,}{ }^{13}$. Some studies have investigated the incidence and the prognostic value of hypoalbuminemia in critically ill children, reporting a higher incidence $(60 \%$ approximately) in cases requiring PICU admission ${ }^{11,16-22}$. In our study, hypoalbuminemia, defined as serum levels less than $3.5 \mathrm{~g} / \mathrm{dl}$, was observed in approximately $15 \%$ of cases of $\mathrm{AB}$ at presentation. There are no clear reference values of serum albumin in children. Therefore, the lower incidence of hypoalbuminemia that we observed could be explained because the albumin level used to define hypoalbuminemia varies widely between the different studies. Also, we did not included patients with previously diagnosed malnutrition, a known facilitating factor for hypoalbuminemia. Furthermore, the inflammatory response in AB, another important determinant of hypoalbuminemia, is usually of lower intensity than in conditions such as cardiac surgery, bacterial pneumonia or sepsis, that were the most frequent diseases included in those studies ${ }^{23}$.

The presence of hypoalbuminemia has been previously shown as a significant marker of mortality and increased stay in children admitted to pediatric intensive care unit ${ }^{11,16-22}$, but to our knowledge this is the second study that have investigated the role of hypoalbuminemia as biomarker for severity in children with $\mathrm{AB}$. Mansbach et al. studied 1016 infants with $\mathrm{AB}$ and found that those cases of AB presenting with hypoalbuminemia had a four-fold risk to develop apnea during hospitalization ${ }^{14}$. Results consistent with aforementioned study were found in the present study. Thus, we found a four-fold risk to present a severe illness in infants with $\mathrm{AB}$ and serum albumin less than $3.5 \mathrm{~g} / \mathrm{dl}$ at presentation. Also, we found that lower levels of serum albumin were correlated with a prolonged LOS hospitalization. It is noteworthy that the incidence of hypoalbuminemia in the study of Mansbach et al. (38\%) was higher than the incidence that we found (16\%). This could be explained by the different cut-off that we used to define hypoalbuminemia $(3.5 \mathrm{~g} / \mathrm{dl}$ vs $3.8 \mathrm{~g} / \mathrm{dl})$. 
Using the same definition of hypoalbuminemia $(3.8 \mathrm{~g} / \mathrm{dl})$, the incidence in our population would raise up to $28 \%$ (data not shown). Despite our differences, the incidence of hypoalbuminemia seems to be lesser in AB than conditions with a more important inflammatory component, as mentioned previously.

The discriminatory value of serum albumin level for predicting PICU admission in our study was fair (AUC ROC 0.70 ), and sensitivity and specificity were relatively low ( $71 \%$ and $68 \%$ respectively) for hypoalbuminemia $(<3.5 \mathrm{~g} / \mathrm{dl})$. However, based on the very high NPV observed $(92 \%)$, serum albumin levels equal or higher than $3.5 \mathrm{~g} / \mathrm{dl}$ could help clinicians to identify those infants with $\mathrm{AB}$ at lower risk for developing a severe disease. The advantage of using of serum albumin levels for predicting outcomes is that it would be an objective, inexpensive and widely available biomarker to use in conjunction with current clinical scores to assess disease severity.

The mechanisms of hypoalbuminemia in critical illness is complex and may involve a number of mechanisms such as an imbalance between albumin synthesis and degradation, increased capillary leakage, and altered intravascular and tissue albumin distribution ${ }^{9,10}$. Interestingly, the presence of hypoalbuminemia seems to be associated with a severe illness independently of the underlying respiratory process. Thus, we did not document a worse respiratory state at admission in relation with hypoalbuminemia, as the BROSJOD clinical score, $\mathrm{pCO} 2, \mathrm{pH}$, serum bicarbonate and serum lactate levels at admission were not associated to serum albumin levels in the multivariate analysis. In the light of our results, the levels of serum albumin in infants with $\mathrm{AB}$ are associated with the age, the weight for age percentile, and the inflammatory response. Accordingly, the positive correlation observed between the age and the serum albumin level has been previously recognized in neonates and small infants ${ }^{24}$, and malnutrition and inflammation are considered to play a major role in occurrence of hypoalbuminemia ${ }^{9}$. Although it has traditionally been linked to malnutrition and we observed that the weight for age percentile was independently associated to serum albumin levels, hypoalbuminemia is not considered a specific nutritional marker ${ }^{9}$. Thus, serum albumin concentration is influenced also by various non-nutritional factors, impairing its validity as a nutritional parameter in patients who have acute-phase response and metabolic stress. Furthermore, a decrease in serum albumin concentration develops usually late in the course of malnutrition and, as a consequence, in the most severe cases that were excluded for this study. Therefore, we think that low serum albumin levels may not be due to the nutritional status of our patients.

Conversely, we think that inflammation could play a key role in the occurrence of hypoalbuminemia in AB. Albumin is assumed as a negative acute phase protein. Inflammation mediated by cytokines leads to decreased synthesis of albumin and causes albumin redistribution associated with increased capillary permeability ${ }^{9,10}$. The multivariate linear regression showed an independent inverse association between CRP and albumin levels, suggesting that hypoalbuminemia in $\mathrm{AB}$ is associated with the acute inflammatory response. The association of low serum albumin levels with high concentrations of inflammatory markers such as CRP, or IL-6 has been previously demonstrated in adults ${ }^{25-27}$. Of note, among the inflammatory mediators that have been described to play an essential role in the Respiratory Syncytial Virus pathology are cytokines ${ }^{6,28}$. Among these, the pro-inflammatory cytokine IL-6 is has been described to be critical for regulating disease severity during RSV infection in mice models, and has been related with the development of neurologic alterations in infants ${ }^{28-30}$. Thus, it could be possible that the increase of inflammatory mediators such as IL6 during $\mathrm{AB}$ leads to an increase in the hepatic production of positive acute phase reactants, such as CRP, while conversely decreasing the production of negative acute phase reactants such as albumin. Because, measurement of IL-6 is usually not readily available and expensive, serum albumin could serve as a low-cost and easily measured biochemical parameter of inflammation in $\mathrm{AB}$.

Limitations: The study has some limitations. This is an observational relatively small size and single centre study; therefore it may not be representative of outcomes at other sites because of special circumstances such as physician quality, hospital features, different resources or hospitalization/PICU admission criteria. A control group was not used to establish normal reference levels of serum albumin. We used the 25th percentile as an arbitrary definition of hypoalbuminemia. We used the initial serum albumin level measured when patients presented to the ED. Strength of this approach was that the initial serum albumin level was 
unlikely to be affected by fluid resuscitation or other possible treatment during ED. However, we did not investigate the trends in serum albumin level, which could reveal more kinetic information and should be a subject of the further research. Although we attempted to collect extensive covariates data and avoid introducing potential bias, unknown confounding factors may have influenced the associations between the predictive and outcome variables.

Conclusion: An observational correlation has been observed between severity of illnessas determined by PICU admission and serum albumin levels; the children who had higher severity of illness had lower albumin levels at hospital admission. Specifically, serum albumin levels higher than $3.5 \mathrm{~g} / \mathrm{dl}$ could help clinicians to identify those infants with $\mathrm{AB}$ at lower risk of develop a severe illness. Hypoalbuminemia in patients with $\mathrm{AB}$ seems to be associated to the acute inflammatory response of the disease. The results of this study suggest that inflammation has a role in severity of illness in the setting of AB.

\section{Acknowledgements:}

This work has been awarded the Best Clinical Research Annual Prize grated by Cadiz's Medical Association.

\section{References}

1. Bont L, Checchia PA, Fauroux B, Figueras-Aloy J, Manzoni P, Paes B, et al. Defining the Epidemiology and Burden of Severe Respiratory Syncytial Virus Infection Among Infants and Children in Western Countries. Infectious Diseases and Therapy. Springer Healthcare; 2016 Aug 1;5(3):271-98.

2. Ghazaly M, Nadel S. Characteristics of children admitted to intensive care with acute bronchiolitis. Eur J Pediatr. 2018 Jun;177(6):913-20.

3. Manzoni P, Figueras-Aloy J, Simões EAF, Checchia PA, Fauroux B, Bont L, et al. Defining the Incidence and Associated Morbidity and Mortality of Severe Respiratory Syncytial Virus Infection Among Children with Chronic Diseases. Infectious Diseases and Therapy. 2017 Sep;6(3):383-411.

4. Ralston SL, Lieberthal AS, Meissner HC, Alverson BK, Baley JE, Gadomski AM, et al. Clinical practice guideline: the diagnosis, management, and prevention of bronchiolitis. Vol. 134, Pediatrics. American Academy of Pediatrics; 2014. pp. e1474-502.

5. Fernandes RM, Plint AC, Terwee CB, Sampaio C, Klassen TP, Offringa M, et al. Validity of bronchiolitis outcome measures. Pediatrics. American Academy of Pediatrics; 2015 Jun;135(6):e1399-408.

6. Brown PM, Schneeberger DL, Piedimonte G. Biomarkers of respiratory syncytial virus (RSV) infection: specific neutrophil and cytokine levels provide increased accuracy in predicting disease severity. Paediatric Respiratory Reviews. 2015 Sep;16(4):232-40.

7. Rodriguez-Gonzalez, Perez-Reviriego, Castellano-Martinez, Lubian-Lopez, Benavente-Fernandez. Left Ventricular Dysfunction and Plasmatic NT-proBNP Are Associated with Adverse Evolution in Respiratory Syncytial Virus Bronchiolitis. Diagnostics. 2019 Sep;9(3):85-15.

8. Rodriguez-Gonzalez M, Benavente Fernández I, Castellano-Martinez A, Lechuga-Sancho AM, LubiánLópez SP. NT-proBNP plasma levels as biomarkers for pulmonary hypertension in healthy infants with respiratory syncytial virus infection. Biomarkers Med. Future Medicine Ltd London, UK; 2019 Jun 3;2017(3):7074508-618.

9. Eckart A, Struja T, Kutz A, Baumgartner A, Baumgartner T, Zurfluh S, et al. Relationship of Nutritional Status, Inflammation, and Serum Albumin Levels During Acute Illness: A Prospective Study. Am J Med. 2019 Nov 18.

10. Don BR, Kaysen G. Serum albumin: relationship to inflammation and nutrition. Semin Dial. John Wiley \& Sons, Ltd; 2004 Nov;17(6):432-7.

11. Durward A, Mayer A, Skellett S, Taylor D, Hanna S, Tibby SM, et al. Hypoalbuminaemia in critically ill children: incidence, prognosis, and influence on the anion gap. Arch Dis Child. BMJ Publishing Group 
Ltd; 2003 May;88(5):419-22.

12. Yap FHY, Joynt GM, Buckley TA, Wong ELY. Association of serum albumin concentration and mortality risk in critically ill patients. Anaesth Intensive Care. 2002 Apr;30(2):202-7.

13. Goldwasser P, Feldman J. Association of serum albumin and mortality risk. J Clin Epidemiol. 1997 Jun;50(6):693-703.

14. Mansbach JM, Geller RJ, Hasegawa K, Espinola JA, Stevenson MD, Sullivan AF, et al. Association of Serum Albumin With Apnea in Infants With Bronchiolitis. JAMA Netw Open. 2019 Jul 17;2(7):e197100-9.

15. Balaguer M, Alejandre C, Vila D, Esteban E, Carrasco JL, Cambra FJ, et al. Bronchiolitis Score of Sant Joan de Déu: BROSJOD Score, validation and usefulness. PediatrPulmonol. 2nd ed. John Wiley \& Sons, Ltd; 2016 Nov 7;52(4):533-9.

16. Yanni GN, Lubis M, Ali M. The Influence of Albumin Level in Critically Ill Children to Length of Stay and Mortality in Paediatric Intensive Care Unit. Open Access Maced J Med Sci. 2019 Oct 30;7(20):3455-8.

17. Henry BM, Borasino S, Ortmann L, Figueroa M, Rahman AKMF, Hock KM, et al. Perioperative serum albumin and its influence on clinical outcomes in neonates and infants undergoing cardiac surgery with cardiopulmonary bypass: a multi-centre retrospective study. Cardiol Young. Cambridge University Press; 2019 Jun;29(6):761-7.

18. Leite HP, Rodrigues da Silva AV, de Oliveira Iglesias SB, Koch Nogueira PC. Serum Albumin Is an Independent Predictor of Clinical Outcomes in Critically Ill Children. Pediatric Critical Care Medicine. 2016 Feb;17(2):e50-7.

19. Kittisakmontri K, Reungrongrat S, Lao-Araya M. Hypoalbuminaemia at admission predicts the poor outcomes in critically ill children. Anaesthesiol Intensive Ther. Termedia; 2016;48(3):158-61.

20. Tiwari LK, Singhi S, Jayashree M, Baranwal AK, Bansal A. Hypoalbuminemia in critically sick children. Indian J Crit Care Med. 2014 Sep;18(9):565-9.

21. Bhandarkar N, Save S, Bavdekar SB, Sisodia P, Desai S. Serum Albumin and C-Reactive Protein as Predictors of Adverse Outcomes in Critically Ill Children: A Prospective Observational Pilot Study. Indian J Pediatr. Springer India; 2019 Aug;86(8):758-9.

22. Horowitz IN, Tai K. Hypoalbuminemia in critically ill children. Arch Pediatr Adolesc Med. American Medical Association; 2007 Nov;161(11):1048-52.

23. Moreno-Solís G, Torres-Borrego J, la Torre-Aguilar de MJ, Fernández-Gutiérrez F, Llorente-Cantarero FJ, Pérez-Navero JL. Analysis of the local and systemic inflammatory response in hospitalized infants with respiratory syncitial virus bronchiolitis. Allergologia et Immunopathologia. 2015 May;43(3):264-71.

24. Torer B, Hanta D, Yapakci E, Gokmen Z, Parlakgumus A, Gulcan H, et al. Association of Serum Albumin Level and Mortality in Premature Infants. J Clin Lab Anal. John Wiley \& Sons, Ltd; 2016 Nov;30(6):867-72.

25. Gibson DJ, Hartery K, Doherty J, Nolan J, Keegan D, Byrne K, et al. CRP/Albumin Ratio: An Early Predictor of Steroid Responsiveness in Acute Severe Ulcerative Colitis. J Clin Gastroenterol. 2018 Jul;52(6):e48-e52.

26. Aksu U, Gulcu O, Aksakal E, Kalkan K, Öztürk M, Korkmaz AF, et al. The association between CRP / Albumin ratio and in-stent restenosis development in patients with ST-segment elevation myocardial infarction. J Clin Lab Anal. John Wiley \& Sons, Ltd; 2019 May;33(4):e22848.

27. Hizli O, Cayir S, Coluk Y, Kayabasi S, Yildirim G. The novel indicators of moderate to severe sleep apnea: fibrinogen to albumin ratio vs. CRP to albumin ratio. Eur Arch Otorhinolaryngol. Springer Berlin Heidelberg; 2020 Jan 9;21(1):37-5. 
28. Bohmwald K, Gálvez NMS, Canedo-Marroquín G, Pizarro-Ortega MS, Andrade-Parra C, GómezSantander F, et al. Contribution of Cytokines to Tissue Damage During Human Respiratory Syncytial Virus Infection. Front Immunol. 2019;10:452.

29. Piedra F-A, Mei M, Avadhanula V, Mehta R, Aideyan L, Garofalo RP, et al. The interdependencies of viral load, the innate immune response, and clinical outcome in children presenting to the emergency department with respiratory syncytial virus-associated bronchiolitis. Varga SM, editor. PLOS ONE. 2017;12(3):e0172953.

30. Vázquez Y, González L, Noguera L, González PA, Riedel CA, Bertrand P, et al. Cytokines in the Respiratory Airway as Biomarkers of Severity and Prognosis for Respiratory Syncytial Virus Infection: An Update. Front Immunol. Frontiers; 2019;10:1154.

\section{Hosted file}

Tables.Albumin.V2.1.pdf available at https://authorea.com/users/281515/articles/488864association-of-serum-albumin-levels-with-inflammation-and-clinical-outcomes-in-childrenwith-acute-bronchiolitis

\section{Hosted file}

Figure legends.pdf available at https://authorea.com/users/281515/articles/488864association-of-serum-albumin-levels-with-inflammation-and-clinical-outcomes-in-childrenwith-acute-bronchiolitis 